Please cite this article as: Demirer and Landry, (2021). Efficient Transient Gene Knock-down in Tobacco Plants Using Carbon Nanocarriers,Bio-protocol

\title{
Efficient Transient Gene Knock-down in Tobacco Plants Using Carbon Nanocarriers
}

Gozde S. Demirer ${ }^{1, \$, *}$ and Markita P. Landry ${ }^{1,2,3,4, *}$

${ }^{1}$ Department of Chemical and Biomolecular Engineering, University of California, Berkeley, CA 94720, USA; ${ }^{2}$ California Institute for Quantitative Biosciences, QB3, University of California, Berkeley, CA 94720, USA; ${ }^{3}$ Innovative Genomics Institute, Berkeley, CA 94702, USA; ${ }^{4}$ Chan-Zuckerberg Biohub, San Francisco, CA 94158, USA; \$Current address: Department of Plant Biology and Genome Center, University of California, Davis, 451 Health Sciences Drive, Davis, CA 95616, USA

*For correspondence: gdemirer@berkeley.edu; landry@berkeley.edu

[Abstract] Gene knock-down in plants is a useful approach to study genotype-phenotype relationships, render disease resistance to crops, and enable efficient biosynthesis of molecules in plants. Small interfering RNA (siRNA)-mediated gene silencing is one of the most common ways to achieve gene knock-down in plants. Traditionally, siRNA is delivered into intact plant cells by coding the siRNA sequences into DNA vectors, which are then delivered through viral and/or bacterial methods. In this protocol, we provide an alternative direct delivery method of siRNA molecules into intact plant cells for efficient transient gene knock-down in model tobacco plant, Nicotiana benthamiana, leaves. Our approach uses one dimensional carbon-based nanomaterials, single-walled carbon nanotubes (SWNTs), to deliver siRNA, and does not rely on viral/bacterial delivery. The distinct advantages of our method are i) there is no need for DNA coding of siRNA sequences, ii) this abiotic method could work in a broader range of plant species than biotic methods, and iii) there are fewer regulatory complications when using abiotic delivery methods, whereby gene silencing is transient without permanent modification of the plant genome.

\section{Graphic abstract:}

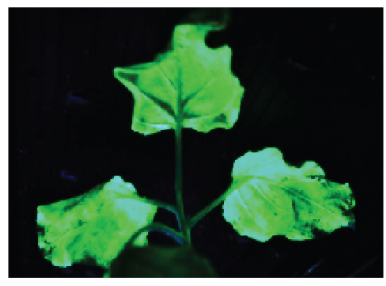

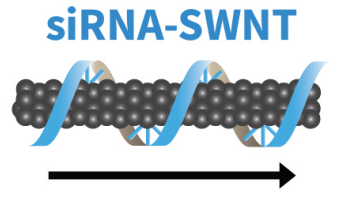

Gene

knock-down

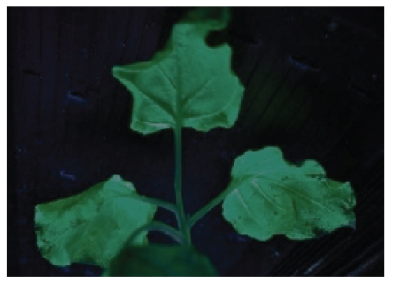

Keywords: Plant gene silencing, RNA interference, siRNA delivery, Gene knock-down, Nicotiana benthamiana, Carbon nanotubes, Single-walled carbon nanotubes (SWNT), Nanomaterials

[Background] Gene silencing through RNA interference (RNAi) was discovered in the early 1990s by plant researchers studying petunia flower coloring (Van der Krol et al., 1990). In RNAi, specifically in post-transcriptional gene silencing (PTGS), gene expression level is reduced through mRNA degradation caused by small RNA molecules - micro (miRNA) or small interfering (siRNA) RNA. RNAi 
has been a breakthrough technology, not only in plant research and biotechnology applications, but also for many other organisms, including human therapy applications (Sierakowska et al., 1996).

The first step of siRNA-mediated RNAi in plants is the delivery of siRNA molecules into plant cells. Delivery is a big bottleneck in plant biotechnology, given the presence of plant cell wall that acts as a physical barrier for the delivery of biotechnology-relevant cargoes such as DNA, RNA, and protein. In plants, siRNA delivery is most commonly accomplished through viral vector delivery via Agrobacterium tumefaciens. However, most plant viruses are limited in their host range (Silva et al., 2010) and the size of cargo they can efficiently deliver (Burch-Smith et al., 2004). Agrobacterium-mediated delivery is also limited in terms of plant host species, causes uncontrolled DNA integration into the plant nuclear genome, and results in constitutive expression of siRNA, which limits temporal control over gene silencing (Baltes et al., 2017).

Carbon nanotubes are one dimensional high-aspect-ratio nanomaterials that have many advantageous features for siRNA delivery in plants. First, given their needle-like structure with a small diameter $(\sim 1 \mathrm{~nm})$, long length $(\sim 500 \mathrm{~nm})$ and high stiffness, single-walled carbon nanotubes (SWNTs) have shown to transport across the plant cell wall and localize inside plant cells (Demirer et al., 2019b). Second, high surface area and diverse surface chemistry options of SWNTs enable delivery of diverse biological cargoes (Beyene et al., 2016; Del Bonis-O'Donnell et al., 2017; Demirer et al., 2020). Lastly, SWNTs have the ability to delay the intracellular degradation of biomolecular cargoes (Demirer et al., 2019a and 2020), which is especially valuable when working with fragile molecules like RNA.

Recently, we have developed a method to deliver siRNA molecules targeting the silencing of a transgenic GFP gene in Nicotiana benthamiana leaves, and an endogenous stress gene, ROQ1, using SWNTs (Demirer et al., 2020). In this approach, we first load sense and antisense strands of siRNA onto two separate SWNT nanoparticle solutions via pi-pi interactions that form between the $\mathrm{sp}^{2}$ carbon nanotube surface lattice and the aromatic bases of single stranded RNA (ssRNA). Next, we introduce an equimolar mixture of these RNA-SWNT solutions into intact plant leaves for GFP silencing. Our results demonstrate efficient silencing of GFP as assessed by confocal microscopy imaging, quantitative PCR (qPCR), and Western blotting, both for transgenic GFP and also for the endogenous ROQ1 gene, with disease-resistance applications (Demirer et al., 2020). This transient gene knock-down approach could be applied to other plant species, tissues, and target genes with minimal modifications. Additionally, the RNA loading method used in this study is not specific to siRNA, and thus, it can be adapted for the delivery of other types of nucleic acids with some optimization (e.g., guide RNA or messenger RNA for CRISPR genome editing applications).

Below, we provide a step-by-step protocol for the synthesis and characterization of siRNA loaded SWNTs, and the measurement of gene silencing efficiency in tobacco leaves through confocal imaging, qPCR and Western blotting (Figure 1). 


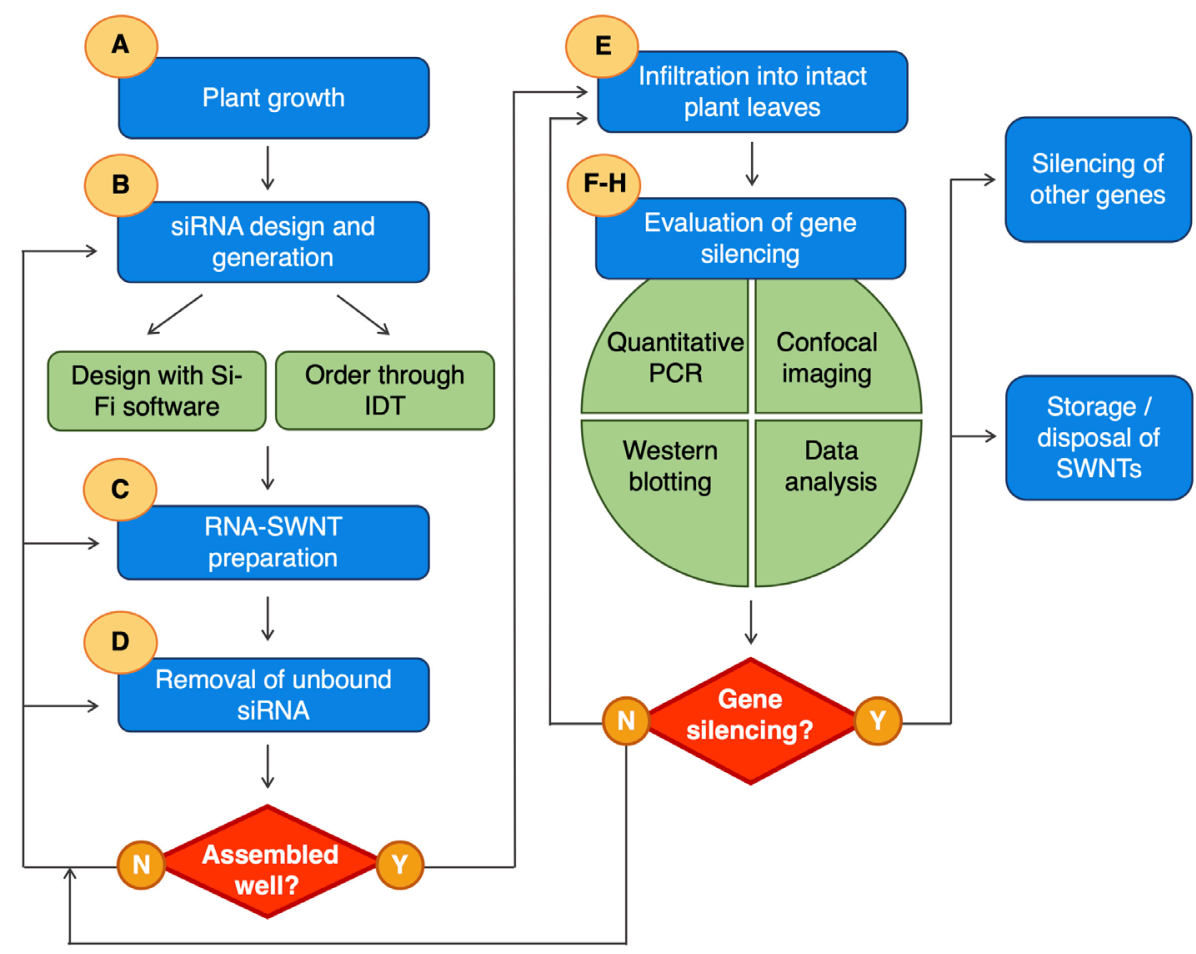

Figure 1. Overview of the siRNA-SWNT gene silencing procedure

\section{Materials and Reagents}

1. SunGro Sunshine LC1 Grower soil mix (SUN52128CFLP)

2. Delicate task wipes (Kimberly-Clark, catalog number: 06-666)

3. 100K MWCO Amicon spin filters (MilliporeSigma, catalog number: UFC510024)

4. PVDF Membrane, Precut, $7 \times 8.4 \mathrm{~cm}$ (Bio-Rad, catalog number: 1620174)

5. Sterile syringe filter $(0.45 \mu \mathrm{m}$; VWR, catalog number: $28145-481)$

6. Microcentrifuge tubes (1.5 ml; VWR, catalog number: 89000-028)

7. Conical tubes (50 ml; Olympus, catalog number: 28-106)

8. Pipette tips (Low retention $10 \mu \mathrm{l}, 200 \mu \mathrm{l}, 1,000 \mu \mathrm{l}$ filter tips; USA Scientific, catalog numbers: 1181-3710, 1180-8710, 1182-1730)

9. Extended-length pipette tips (1,000 $\mu$; Eppendorf, catalog number: 0030073614)

10. \#1 Microscopy cover glass (Fisher Scientific, catalog number: 12-542B)

11. Microscope slides (VWR, catalog number: 16004-422)

12. Syringe ( $1 \mathrm{ml} ; \mathrm{BD}$, catalog number: $14-823-434)$

13. Mini Trans-Blot Filter paper (Bio-Rad, catalog number: 1703932)

14. EasyStrip, Plus PCR Tube (Thermo Scientific, catalog number: AB2005)

15. Plant seeds ( $m$ GFP5 Nicotiana benthamiana is obtained from the Staskawicz lab at UC Berkeley, $m G F P 5$ plants constitutively express GFP targeted to the ER under the control of the Cauliflower mosaic virus $35 \mathrm{~S}$ promoter) 
16. Goat anti-rabbit horseradish peroxidase-conjugated antibody (Abcam, catalog number: ab205718)

17. Anti-GFP antibody, ChIP Grade (Abcam, catalog number: ab290)

18. HiPCO SWNTs (Nanolntegris, Super purified, catalog number: HS28-037)

19. MilliQ water

20. Nuclease-free water (Qiagen, catalog number: 129114)

21. Sodium chloride, $\mathrm{NaCl}$ (Sigma-Aldrich, catalog number: S9888-500G)

22. Hydrochloric acid, $\mathrm{HCl}$ (37\% [vol/vol]; Sigma, catalog number: 320331)

23. Single-stranded RNA oligonucleotides, including sense and antisense siRNA strands - 21 nucleotides (Integrated DNA Technologies, IDT)

24. Sodium dodecyl sulfate, molecular biology grade (Sigma-Aldrich, catalog number: 436143100G)

25. Tris/HCl (Sigma-Aldrich, catalog number: 10812846001)

26. EDTA (Sigma-Aldrich, catalog number: E9884-100G)

27. NP-40 (Sigma-Aldrich, catalog number: 492016-100ML)

28. Glycerol (Sigma-Aldrich, catalog number: G5516-500ML)

29. Pierce $660 \mathrm{~nm}$ Protein Assay (Thermo, catalog number: 22660)

30. iScript cDNA synthesis kit (Bio-Rad, catalog number: 1708891)

31. PowerUp SYBR green master mix (Applied Biosystems, catalog number: A25742)

32. Qubit Protein Assay (ThermoFisher Scientific, catalog number: Q33211)

33. RNeasy plant mini kit (QIAGEN, catalog number: 74904)

34. BSA (Sigma-Aldrich, catalog number: A4737-25G)

35. TWEEN20 (Sigma-Aldrich, catalog number: P9416-100ML)

36. Ammonium persulphate, APS (Sigma, catalog number: 248614-100G)

37. Low range ultra agarose (Bio-Rad, catalog number: 1613107)

38. ECL Prime Western Blotting System (MilliporeSigma, catalog number: GERPN2232)

39. TEMED (N,N,N,N'-tetramethylethylenediamine; Sigma, catalog number: T9281)

40. Glycine (Sigma, catalog number: G8898)

41. Methanol (Sigma, catalog number: 179957)

42. $4 \times$ Laemmli sample Buffer (Bio-Rad, $10 \mathrm{ml}$, catalog number: 1610747)

43. Liquid nitrogen

44. SYBR Gold Nucleic Acid Gel Stain (Invitrogen, catalog number: S11494)

45. $30 \%$ Acrylamide/Bis solution 19:1 (Bio-Rad, catalog number: 1610154)

46. Protease inhibitor cocktail (Sigma, catalog number: P9599-1ML)

47. $0.1 \mathrm{M} \mathrm{NaCl}$ (see Recipes)

48. $10 \%$ (wt/vol) Ammonium persulphate solution (APS) (see Recipes)

49. $10 \times$ Transfer buffer (see Recipes)

50. $1 \times$ Transfer buffer (see Recipes)

51. $10 \times$ Tris-Buffered Saline (TBS) buffer (1 M Tris, $1.5 \mathrm{M} \mathrm{NaCl}, \mathrm{pH} 7.4$ ) (see Recipes) 
52. $1 \times$ TBST buffer (see Recipes)

53. Lysis buffer (see Recipes)

\section{Equipment}

1. Analytical balance (Radwag, model: AS 60/220.R2)

2. Ultrasonic bath (Branson, model: 15-336-100)

3. Ultrasonic homogenizer with 6-mm tip (Cole-Parmer, models: UX-04711-70, UX-04712-14)

4. Vortex mixer (Fisher Scientific, model: 02-215-365)

5. $\mathrm{pH}$ meter (Spectrum, model: 242-97839)

6. Orbital shaker (Waverly, model: S1CE)

7. NanoVue Plus spectrophotometer (GE Life Sciences, model: 28-9569-61)

8. Visible spectrophotometer (Thermo Scientific, model: 14-385-445)

9. Near-infrared spectrometer (Princeton Instruments IsoPlane 320 coupled to a liquid nitrogencooled Princeton Instruments PyLoN-IR 1D array of InGaAs pixels)

10. UV-Vis-NIR Spectrophotometer (Shimadzu, model: UV-3600 Plus)

11. Tabletop centrifuge (Eppendorf, catalog number: 5418000017)

12. Centrifuge (Eppendorf, model: $5424 \mathrm{R})$

13. Tweezers (VWR, catalog number: 63042-518)

14. Scissors (VWR, catalog number: 82027-582)

15. Mortar and pestle (Cole-Parmer, catalog number: EW-63100-54)

16. Plant growth chamber (HiPoint, model: 740 FHLED)

17. Gel image-analysis system (Typhoon FLA 9500, GE Healthcare Services)

18. Electrophoresis power supply (PowerPac basic power supply; Bio-Rad, catalog number: 1645050)

19. Mini Trans-Blot Cell (Bio-Rad, catalog number: 1703811)

20. Mini-Protein TGX gels (Bio-Rad, catalog number: 456-1094)

21. ChemiDoc XRS+ System (Bio-Rad, catalog number: 1708265)

22. Confocal Microscope (Zeiss, model: LSM 710)

23. Thermal Cycler CFX96 Touch Real-Time PCR Detection System (Bio-Rad, catalog number: 1855195)

24. Thermal Cycler PCR (Applied Biosystems Veriti 96-Well, catalog number: 4375786)

\section{Software}

1. GraphPad Prism 7.0a (https://www.graphpad.com/scientific-software/prism/)

2. Fiji ImageJ 2.0.0 (https://imagej. net/Fij//Downloads)

3. Zen Blue 2.6 (https://www.zeiss.com/microscopy/us/downloads. html) 


\section{Procedure}

A. Plant growth

Germinate transgenic mGFP5 Nicotiana benthamiana seeds (see Note 1) and grow seeds in SunGro Sunshine LC1 Grower soil mix in a growth chamber for four to six weeks before experiments. Use $12 \mathrm{~h}$ light at $24{ }^{\circ} \mathrm{C}$ and $12 \mathrm{~h}$ dark at $18^{\circ} \mathrm{C}$ cycles for growing plants.

Note: Different plant species may require different germination and growth conditions.

B. siRNA design and generation

1. Currently, there are many software programs to design gene specific siRNA sequences with minimal off-target effects. A recently developed software called "siRNA-Finder (si-Fi) Software" can be used in plants (Lück et al., 2019).

2. After the design of siRNA sequences, sense and antisense RNA strands can be purchased from Integrated DNA Technologies (IDT) as single-stranded oligonucleotides.

C. RNA-SWNT preparation

1. Dissolve sense and antisense siRNA strands in $0.1 \mathrm{M} \mathrm{NaCl}$ at a concentration of $100 \mathrm{mg} / \mathrm{ml}$.

2. Add $1 \mathrm{mg}$ dry HiPCO SWNTs to $20 \mu \mathrm{l}$ of dissolved sense RNA, and complete the solution volume to $1 \mathrm{ml}$ with $0.1 \mathrm{M} \mathrm{NaCl}$ (see Note 2).

3. Bath sonicate the mixture for $10 \mathrm{~min}$ at room temperature in Ultrasonic bath (Branson).

4. Probe-tip sonicate the mixture with a $3 \mathrm{~mm}$ tip at $50 \%$ amplitude $(\sim 7 \mathrm{~W})$ for $30 \mathrm{~min}$ in an ice bath. Renew ice bath if it starts melting during the sonication to prevent heating.

5. Rest the solution at room temperature for $30 \mathrm{~min}$.

6. Centrifuge the solution at $16,100 \times g$ for $1 \mathrm{~h}$ in room temperature to remove bundled SWNTs. The supernatant contains the individually suspended sense-RNA-SWNTs. Keep the supernatant and discard the SWNT pellet to the hazardous nanomaterials waste.

7. Repeat the same protocol for the antisense RNA strand (see Note 3). Store RNA-SWNT solutions at $4{ }^{\circ} \mathrm{C}$.

D. Removal of unbound siRNA

1. Add $500 \mu \mathrm{l}$ sense-RNA-SWNT and $500 \mu \mathrm{l}$ antisense-RNA-SWNT into two separate $100 \mathrm{~K}$ Amicon spin filters that are placed in $2 \mathrm{ml}$ collection tubes. Centrifuge $4 \mathrm{~min}$ at $8,000 \times \mathrm{g}$ in room temperature.

2. Collect the flow-throughs from sense-RNA-SWNT and antisense-RNA-SWNT in separate tubes and place the spin filters into the same collection tubes.

3. Add $0.1 \mathrm{M} \mathrm{NaCl}$ into the spin filters until the volume reaches $500 \mu \mathrm{l}$. Repeat the wash step.

4. Perform a total of 8 washes to remove all unbound RNA molecules. Merge and accumulate all flow-through solutions (separately for sense-RNA-SWNT and antisense-RNA-SWNT) for later measurement of removed RNA amount. 
5. Calculate the SWNT concentration by measuring the carbon nanotube absorbance at $632 \mathrm{~nm}$ using a spectrophotometer (use $2 \mu \mathrm{l}$ for NanoVue Plus spectrophotometer or dilute to $1 \mathrm{ml}$ for Thermo Scientific). Divide the absorbance value by SWNT extinction coefficient of 0.036 to obtain SWNT concentration in the unit of $\mu \mathrm{g} / \mathrm{ml}$ (If the sample is diluted, multiply the absorbance value also by the dilution factor).

6. Calculate the concentration of RNA loaded on SWNTs by measuring the absorbance of collected flow-through solutions at $260 \mathrm{~nm}$ (use $2 \mu \mathrm{l}$ for NanoVue Plus spectrophotometer), and subtracting the total amount of RNA removed from the total amount of RNA added ( $2 \mathrm{mg}$ in this case).

7. (Optional) For additional characterization, record sense-RNA-SWNT and antisense-RNASWNT absorbance spectra with Shimadzu UV-3600 Plus, and fluorescence spectra with a nearinfrared spectrometer (Princeton Instruments IsoPlane 320 coupled to a liquid nitrogen-cooled Princeton Instruments PyLoN-IR 1D array of InGaAs pixels). See Demirer et al., 2020 for representative examples of SWNT absorbance and fluorescence spectra.

E. Infiltration of leaves with RNA-SWNTs

1. Select healthy and fully-developed leaves from mGFP5 Nicotiana benthamiana (4-6 weeks old) plants for experiments.

2. Merge $100 \mu \mathrm{l}$ of $200 \mathrm{nM}$ sense-RNA-SWNTs with $100 \mu \mathrm{l}$ of $200 \mathrm{nM}$ antisense-RNA-SWNTs in a $1.5 \mathrm{ml}$ Eppendorf tube (see Note 4). Mix well.

3. Immediately after mixing, make a small puncture on the abaxial (bottom) surface of the leaf with a pipette tip, and infiltrate $\sim 100-200 \mu \mathrm{l}$ of the siRNA-SWNT mixture from the hole with a $1 \mathrm{ml}$ needleless syringe with caution not to damage the leaf (see Note 5).

4. Use a Kimwipe tissue to remove the excess siRNA-SWNT solution on the leaf surface. Mark the infiltrated area with a Sharpie pen without damaging the leaf.

5. Infiltrate a negative control solution, such as the free siRNA without SWNTs or scrambled RNA suspended SWNTs that does not target the gene of interest. If possible, also infiltrate a positive control solution, such as viral siRNA delivery sample (see Note 6).

6. Return the infiltrated plant(s) into the growth chamber until the measurement of gene silencing.

F. Gene silencing determination through quantitative PCR (qPCR)

1. $24 \mathrm{~h}$ after infiltration, cut the infiltrated leaf areas (maximum of $100 \mathrm{mg}$ leaf tissue per sample) and extract total RNA with an RNeasy plant mini kit. After cutting the leaf, immediately proceed with the first step of the RNA extraction protocol (i.e., grinding the tissue in liquid nitrogen using mortar and pestle) to make sure gene expression levels do not change after cutting. Follow the protocol of the RNeasy plant mini kit (see Note 7).

2. Following RNA extraction, measure the RNA concentration and purity with a spectrophotometer. Reverse transcribe $1 \mu \mathrm{g}$ total RNA into complementary DNA (cDNA) using an iScript cDNA synthesis kit. Follow the protocol of the iScript cDNA synthesis kit. 
Please cite this article as: Demirer and Landry, (2021). Efficient Transient Gene Knock-down in Tobacco Plants Using Carbon Nanocarriers,Bio-protocol

3. Use PowerUp SYBR green master mix for the qPCR step with $2 \mu \mathrm{l}$ cDNA from Step 2 and specific primers for the target and housekeeping genes. Follow the protocol of the PowerUp SYBR green kit for relative quantification of target mRNA in the siRNA-SWNT infiltrated leaf compared to negative controls of free siRNA without SWNTs or scrambled RNA-SWNTs.

Example: The target gene in our qPCR was mGFP5 (GFP transgene inserted into Nb), and EF1 (elongation factor 1) as our housekeeping (reference) gene.

4. Analyze the qPCR data using the ddCt method (Rao et al., 2013). See Figure 2B for representative qPCR results.

G. Gene silencing determination through confocal fluorescence microscopy imaging

1. If silencing a fluorescent protein, such as GFP, confocal fluorescence microscopy can be used to detect approximate silencing efficiency.

2. After infiltration, leave the plants with intact infiltrated leaves in the growth chamber for $72 \mathrm{~h}$.

3. $72 \mathrm{~h}$ after infiltration, cut a small flat piece $(0.5-1 \mathrm{~cm} \times 0.5-1 \mathrm{~cm})$ of the infiltrated leaf around the infiltration point and prepare a glass slide with cover slip (thickness \#1). Add 50-100 $\mu$ l water in between the glass slide and cover slip for imaging. Image samples before the leaf piece dries out (optimally within 15 to $30 \mathrm{~min}$ ).

4. Image the plant tissue with $488 \mathrm{~nm}$ laser excitation with a GFP filter cube (in the case of GFP silencing), and also capture brightfield with a transmitted light if available (see Note 8).

5. Capture at least 10 to 15 fields of view with same optical settings per sample, including nontreated leaf, and any negative and positive control samples.

6. For each sample, compare the mean fluorescence intensity value with the mean GFP fluorescence intensity of a non-treated leaf, which can be used to determine silencing efficiency of siRNA-SWNTs. Pay attention to use the same imaging parameters and quantification analyses for samples imaged on different days. See Figure $2 \mathrm{~A}$ for representative confocal imaging results. 
A

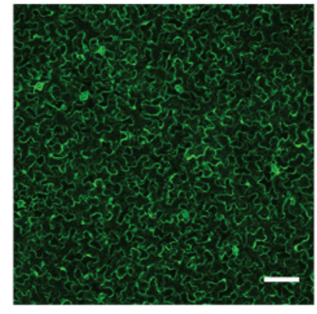

Non-treated

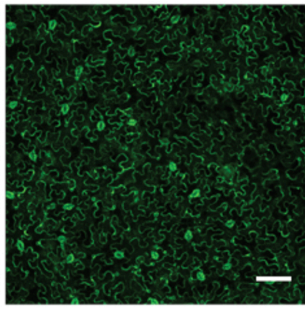

Free siRNA

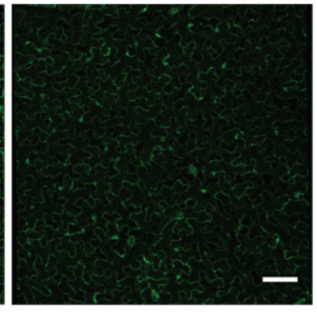

siRNA-SWNT

B

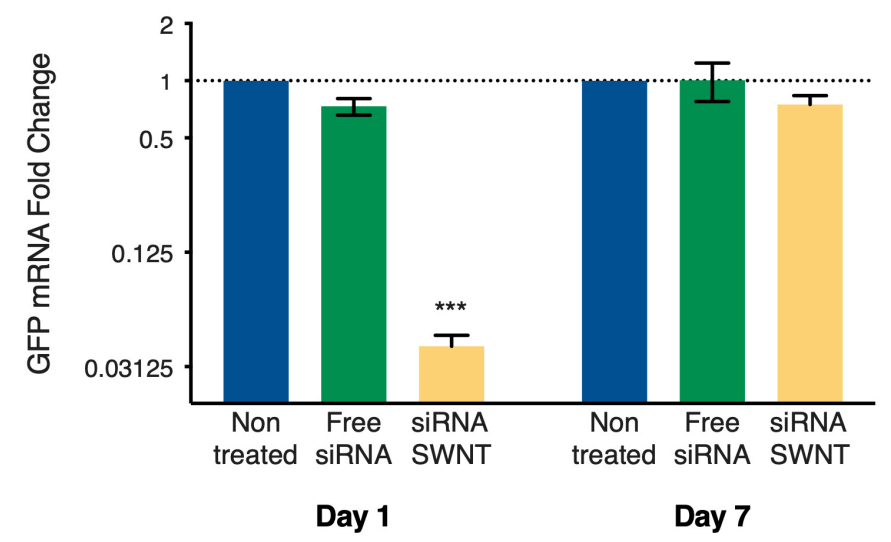

Figure 2. Representative gene silencing results. A. Confocal microscopy images of nontreated and free-siRNA treated control leaves and siRNA-SWNT infiltrated sample leaves of Nicotiana benthamiana. Imaged after 3 days post-infiltration, scale bars are $50 \mu \mathrm{m}$. B. Quantitative PCR analysis results for GFP gene silencing in Nicotiana benthamiana leaves with SIRNA-SWNTS.

H. Gene silencing determination through Western blotting

1. $72 \mathrm{~h}$ after infiltration, harvest infiltrated leaves and grind them in liquid nitrogen using mortar and pestle to recover dry frozen leaf powders.

2. Transfer the frozen leaf powder into a tube with $400 \mu \mathrm{l}$ pre-chilled lysis buffer (see Recipes).

3. Lyse tissue on ice for $1 \mathrm{~h}$. Centrifuge the tubes at $10,000 \times g$ at $4{ }^{\circ} \mathrm{C}$ for $20 \mathrm{~min}$. Following centrifugation, gently transfer the supernatants containing whole proteins to a new clean tube. Quantify total extracted proteins with a Pierce $660 \mathrm{~nm}$ Protein Assay.

4. Mix the samples with the appropriate loading buffer for gel electrophoresis, and boil the mixture at $95-100{ }^{\circ} \mathrm{C}$ for 5 min either using a heat block or thermal cycler. Load $0.5 \mu \mathrm{g}$ of normalized total proteins from each sample and analyze with SDS-PAGE gel (Bio-Rad precast tris/glycine gel, 4-20\% gradient). Run the gel at $120 \mathrm{~V}$ for $60 \mathrm{~min}$.

5. Transfer the gel to a PVDF membrane in cold transfer buffer (see Recipes) and run at $400 \mathrm{~mA}$ in $1 \times$ transfer buffer with methanol for no more than $60 \mathrm{~min}$ in a cold room with an ice block.

6. Block the membrane for $1 \mathrm{~h}$ using $7.5 \%$ BSA in $1 \times$ TBST buffer (see Recipes) followed by overnight incubation at $4{ }^{\circ} \mathrm{C}$ with the primary GFP antibody. After extensive washing, probe the corresponding protein bands with a goat anti-rabbit horseradish peroxidase-conjugated 
antibody for $30 \mathrm{~min}$. After washing, develop the bands by incubation with chemiluminescence (Amersham ECL prime kit) in less than 2 min and image with a ChemiDoc XRS+ System.

7. Quantify the intensity of GFP bands with ImageJ software.

\section{Data analysis}

1. For all experiments, perform at least 3 biological replicates. Biological replicates are defined as experiments consisting of independent infiltrations with RNA-SWNTs into different plants.

2. For quantitative analysis of confocal imaging, capture at least 10-15 non-overlapping fields of view per sample and per biological replicate, which is defined as technical replicates. Average the fluorescence intensity of these technical replicates to obtain the mean fluorescent intensity for a given biological replicate. Confocal images can be analyzed with either the ImageJ or Zen Blue software.

3. For the qPCR assay, perform at least 3 technical replicates, which are defined as reactions from the same isolated RNA batch obtained from the same infiltration. To analyze qPCR results for gene silencing efficiency, used the previously developed ddCt method. The details of this method can be found in Rao et al. (2013).

4. When comparing more than 2 samples with each other statistically for a single independent variable (e.g., silencing efficiency), use one-way ANOVA with Tukey's multiple comparisons test. Report both the $P$ value and $F$ value. When comparing more than 2 samples with each other statistically for multiple independent variables (e.g., silencing efficiency over multiple time points), use two-way ANOVA with Sidak's multiple comparisons test. Report both the $P$ value and $F$ value. When comparing two samples only, Student's $t$-test can be used. Report the $P$ value. GraphPad Prism can be used to plot the data and perform statistical significance analysis.

\section{$\underline{\text { Notes }}$}

1. This protocol is provided for the silencing of GFP gene in transgenic tobacco (Nicotiana benthamiana) leaves, but can be adapted for the silencing of other genes and in other plant species, including other transgenes or endogenous genes.

2. RNA and SWNT amounts can be scaled up or down depending on the experimental need. In scaling, keep the mass ratio of RNA:SWNT at 2. Different final siRNA concentration may be needed for different gene targets, or in different plant species or tissues.

3. Typically, if one RNA strand suspends SWNTs well, its complementary strand has a lower suspension efficiency. This is expected given the complementary base pairs of the sense and antisense strands which can lead to varying adsorption ability of RNA bases to the SWNT surface. We typically obtain 20-50 $\mu \mathrm{g} / \mathrm{ml}$ RNA-SWNT suspensions after centrifugation and purification steps. 
4. Pay attention that $200 \mathrm{nM}$ is the RNA concentration on SWNTs, and not the SWNT concentration. Depending on the suspension efficiency, this corresponds to $\sim 2 \mu \mathrm{g} / \mathrm{ml}$ SWNTs.

5. 100-200 $\mu \mathrm{l}$ infiltration is suggested for confocal imaging studies, whereas larger volume or multiple infiltrations should be performed for qPCR and Western blot to cover the full leaf area.

6. It is preferred to infiltrate the SWNT and control samples on the same leaf to avoid leaf to leaf variation in gene expression and imaging.

7. RNA work must be performed carefully on a clean bench (optimally dedicated to RNA-based experiments) to prevent RNA degradation. Wipe the surfaces and equipment with RNase solution and change gloves frequently. Keep the RNA on ice during the protocol, and transfer to $-20^{\circ} \mathrm{C}$ or $-80{ }^{\circ} \mathrm{C}$ when the protocol is completed.

8. If the leaf is too thick or wavy to get the entire field of view into focus, image with z-stack and perform the silencing analysis on the stacked image. Keep the imaging parameters same between samples that will be compared with each other.

\section{Recipes}

1. $0.1 \mathrm{M} \mathrm{NaCl}$

Weigh $0.5844 \mathrm{~g}$ of $\mathrm{NaCl}$ and dissolve in $100 \mathrm{ml}$ nuclease free water Sterile filter with 0.22-micron syringe filter

2. $10 \%(\mathrm{wt} / \mathrm{vol})$ Ammonium persulphate solution (APS)

Add $5 \mathrm{~g}$ of APS to $50 \mathrm{ml}$ of MilliQ water, and mix to dissolve The solution can be stored at $4{ }^{\circ} \mathrm{C}$ for up to 3 months

3. 10x Transfer buffer

To make $1 \mathrm{~L}$ of $10 \mathrm{x}$ Transfer buffer, add $30.3 \mathrm{~g}$ Tris base, $144 \mathrm{~g}$ Glycine to $800 \mathrm{ml}$ MilliQ water, and mix to dissolve

Then add MilliQ water to a final volume of $1 \mathrm{~L}$

4. $1 \times$ Transfer buffer

To make $1 \mathrm{~L}$ of $1 \times$ Transfer buffer, add $100 \mathrm{ml} \mathrm{10 \times} \mathrm{Transfer} \mathrm{buffer,} 200 \mathrm{ml}$ methanol to $700 \mathrm{ml}$ MilliQ water and mix the solution

The buffer needs to be stored at $4{ }^{\circ} \mathrm{C}$, and it is better to prepare the buffer before running each experiment

5. $10 \times$ Tris-Buffered Saline (TBS) buffer (1 M Tris, $1.5 \mathrm{M} \mathrm{NaCl}, \mathrm{pH} 7.4$ )

To make $250 \mathrm{ml} 10 \times$ TBS buffer, add $30.3 \mathrm{~g}$ Tris base, $21.9 \mathrm{~g} \mathrm{NaCl}$ to $200 \mathrm{ml}$ MilliQ water and mix to dissolve

Use $\mathrm{HCl}$ to adjust the $\mathrm{pH}$ of the solution to 7.4 , then add MilliQ water to a final volume of $250 \mathrm{ml}$ The solution can be stored at $4{ }^{\circ} \mathrm{C}$ for up to 6 months

6. $1 \times$ TBST buffer

Add $50 \mathrm{ml} 10 \times$ TBS buffer, and $500 \mu \mathrm{l}$ of Tween $20(0.1 \%)$ to $500 \mathrm{ml}$ MilliQ water and mix the solution 
The buffer can be stored at $4{ }^{\circ} \mathrm{C}$ for at least 1 month

7. Lysis buffer

$10 \mathrm{mM}$ Tris/HCl

$150 \mathrm{mM} \mathrm{NaCl}$

$1 \mathrm{mM}$ EDTA

$0.1 \% \mathrm{NP}-40$

$5 \%$ glycerol

$1 \%$ protease inhibitor cocktail

$\mathrm{pH} 7.5$

To make $100 \mathrm{ml}$ lysis buffer, add $1.21 \mathrm{~g}$ of Tris base, $0.877 \mathrm{~g} \mathrm{NaCl}, 29.24 \mathrm{mg}$ EDTA, $0.1 \mathrm{~g} \mathrm{NP}$ 40 , and $5 \mathrm{ml}$ glycerol to $80 \mathrm{ml}$ nuclease free water, mix to dissolve, adjust the $\mathrm{pH}$ to $7.5 \mathrm{by} \mathrm{HCl}$, add $1 \mathrm{ml}$ protease inhibitor cocktail, and fill the final volume to $100 \mathrm{ml}$ using the nuclease free water.

Note: The lysis buffer can be stored at $-20^{\circ} \mathrm{C}$ for at least 6 months.

\section{Acknowledgments}

This protocol is derived from Demirer et al. (2020).

G.S.D. is supported by the Schlumberger Foundation Faculty for the Future Program and Caltech Resnick Sustainability Institute. We acknowledge support of a Burroughs Wellcome Fund Career Award at the Scientific Interface (CASI), a Stanley Fahn PDF Junior Faculty Grant with Award \# PFJFA-1760, a Beckman Foundation Young Investigator Award, a USDA AFRI award, a USDA NIFA award, the Moore Foundation, and an FFAR New Innovator Award (M.P.L). M.P.L. is a ChanZuckerberg Biohub investigator.

\section{Competing interests}

Authors declare no competing interest.

\section{$\underline{\text { References }}$}

1. Baltes, N. J., Gil-Humanes, J. and Voytas, D. F. (2017). Genome Engineering and Agriculture: Opportunities and Challenges. Prog Mol Biol Transl Sci 149: 1-26.

2. Beyene, A. G., Demirer, G. S. and Landry, M. P. (2016). Nanoparticle-Templated Molecular Recognition Platforms for Detection of Biological Analytes. Curr Protoc Chem Biol 8(3): 197223.

3. Burch-Smith, T. M., Anderson, J. C., Martin, G. B. and Dinesh-Kumar, S. P. (2004). Applications and advantages of virus-induced gene silencing for gene function studies in plants. Plant J 39(5): 734-746. 
Please cite this article as: Demirer and Landry, (2021). Efficient Transient Gene Knock-down in Tobacco Plants Using Carbon Nanocarriers,Bio-protocol

4. Del Bonis-O’Donnell, J. T., Beyene, A., Chio, L., Demirer, G., Yang, D. and Landry, M. P. (2017). Engineering Molecular Recognition with Bio-mimetic Polymers on Single Walled Carbon Nanotubes. J Vis Exp 119: e55030.

5. Demirer, G. S., Zhang, H., Goh, N. S., Gonzalez-Grandio, E. and Landry, M. P. (2019a). Carbon nanotube-mediated DNA delivery without transgene integration in intact plants. Nat Protoc 14(10): 2954-2971.

6. Demirer, G. S., Zhang, H., Goh, N. S., Pinals, R. L., Chang, R. and Landry, M. P. (2020). Carbon nanocarriers deliver siRNA to intact plant cells for efficient gene knockdown. Sci Adv 6(26): eaaz0495.

7. Demirer, G. S., Zhang, H., Matos, J. L., Goh, N. S., Cunningham, F. J., Sung, Y., Chang, R., Aditham, A. J., Chio, L., Cho, M. J., Staskawicz, B. and Landry, M. P. (2019b). High aspect ratio nanomaterials enable delivery of functional genetic material without DNA integration in mature plants. Nat Nanotechnol 14(5): 456-464.

8. Lück, S., Kreszies, T., Strickert, M., Schweizer, P., Kuhlmann, M. and Douchkov, D. (2019). siRNA-Finder (si-Fi) Software for RNAi-Target Design and Off-Target Prediction. Front Plant Sci 10: 1023.

9. Rao, X., Huang, X., Zhou, Z. and Lin, X. (2013). An improvement of the $2^{\wedge}$ (-delta delta CT) method for quantitative real-time polymerase chain reaction data analysis. Biostat Bioinforma Biomath 3(3): 71-85.

10. Sierakowska, H., Sambade, M. J., Agrawal, S. and Kole, R. (1996). Repair of thalassemic human $\beta$-globin mRNA in mammalian cells by antisense oligonucleotides. Proc Natl Acad Sci 93(23): 12840-12844.

11. Silva, A. T., Nguyen, A., Ye, C., Verchot, J. and Moon, J. H. (2010). Conjugated polymer nanoparticles for effective siRNA delivery to tobacco BY-2 protoplasts. BMC Plant Biol 10: 291.

12. van der Krol, A. R., Mur, L. A., Beld, M., Mol, J. N. and Stuitje, A. R. (1990). Flavonoid genes in petunia: addition of a limited number of gene copies may lead to a suppression of gene expression. Plant Cell 2(4): 291-299. 